\title{
Violência urbana e saúde mental: desafios de uma nova agenda?
}

\author{
Hérica Cristina Batista Gonçalves, Marcello Roriz de Queiroz, Pedro Gabriel Godinho Delgado` \\ Universidade Federal do Rio de Janeiro, Rio de Janeiro, RJ, Brasil
}

Resumo

O fenômeno da violência produz impactos importantes para o setor saúde, seja como fator de agravo à população, ou como barreira de acesso aos serviços. Este artigo é uma nota preliminar de pesquisa exploratória qualitativa. Faz uma revisão sucinta da literatura sobre violência urbana e agravos psicossociais, descreve e analisa o desenvolvimento das ações de saúde mental do Núcleo de Apoio a Saúde da Família (NASF), num contexto de violência. Conclui que, apesar das limitações, a iniciativa do NASF mostra-se valiosa, como vetor de cuidado à população e aos profissionais de atenção primária, $e$ articulador das estratégias psicossociais no território.

Palavras-chave: violência urbana; Atenção Básica; matriciamento; saúde mental e violência urbana.

\section{Urban violence and mental health: challenges of a new agenda?}

\begin{abstract}
The phenomenon of violence produces severe impact on health sector, as related to the population diseases and suffering as barrier of access for medical services. This article is a preliminary note of explorative qualitative research. The article performed a small literature review about urban violence and its psychosocial consequences, describes and analyses the mental health activities from supervision teams of Family Health Program (NASF) in a context of urban violence. Preliminary conclusions show that, despite limitations, the NASF initiative adds value, as support of care for population and professionals, and as a tool to integrate psychosocial strategies in the territory.
\end{abstract}

Keywords: urban violence; primary care; collaborative care in mental health; mental health and urban violence.

\section{Introdução}

Dentre os desafios e iniciativas da reforma psiquiátrica brasileira está a inserção da saúde mental na atenção básica. Como componente de tal desafio, a questão da violência nas grandes cidades mostra-se um fator determinante no modo como essas equipes desenvolvem estratégias de suporte em seus territórios de referência. Partindo do pressuposto de que a estratégia saúde da família (ESF) eleva à radicalidade os princípios de territorialização das atividades de saúde, este artigo tem como objetivo discutir os desafios impostos pelo contexto da violência urbana às estratégias de saúde mental na atenção primária. Para tanto, serão apresentadas algumas dimensões de vulnerabilidade psicossocial de usuários e profissionais, e as possibilidades de suporte a partir dos profissionais de atenção primária. Este artigo é uma nota preliminar de pesquisa, e inclui alguns resultados qualitativos iniciais de pesquisa em andamento.1 Será explorada a relação entre a dinâmica da violência urbana em uma comunidade da Zona Norte do Rio de Janeiro e as consequências psicossociais para Agentes Comunitários de Saúde e usuários de duas unidades de atenção básica.

\footnotetext{
^Endereço para correspondência: Universidade Federal do Rio de Janeiro, Instituto de Psiquiatria, Nuppsam Núcleo de Pesquisas em Políticas Públicas de Saúde Mental. Av. Venceslau Bras, 71 - Fundos. Botafogo - Rio de Janeiro, RJ Brasil. CEP: 22290-140.E-mail: hericacris@yahoo.com.br, marcellorqueiroz@ yahoo.com.br, p.g.godinhodelgado@gmail.com

'Pesquisa intitulada: Desafios da Saúde Mental frente à violência do território, iniciada no ano de 2013, no NUPPSAM/IPUB/UFRJ. Objetiva sistematizar explorar saberes e práticas no desenvolvimento das ações de saúde mental do Núcleo de Apoio à Saúde da Família (NASF), num contexto de violência armada, sob a ótica dos agentes comunitários de saúde - ACS. Com análise das possibilidades e novas invenções, espera-se que diferentes modos de organização do trabalho em saúde, principalmente para a condução dos casos de saúde mental, possam ser elaborados ou potencializados, juntamente com a avaliação do que vem dando certo, o que não tem funcionado e os motivos para estes êxitos fracassos. Projeto em andamento aprovado pelo comitê de ética: 5263 - Instituto de Psiquiatria da Universidade Federal do Rio de Janeiro/ IPUB/ UFRJ. CAAE: 24496113.7.0000.5263
}

A amostra utilizada na pesquisa foi não sistemática e constituída de treze Agentes Comunitários de Saúde (ACS), um de cada equipe da ESF pertencentes às unidades de exploração- dois e Centros Municipais de Saúde-CMS. As informações foram coletadas pela psicóloga do Núcleo de Apoio à Saúde da Família (NASF), que utilizou roteiro e entrevista individual. O roteiro da entrevista foi semiestruturado, composto de questões abertas e direcionadas para os seguintes temas: estratégias de enfrentamento utilizadas para lidar com o contexto de violência urbana, avaliação do território em meio à violência, papel do NASF neste contexto e possíveis sugestões para melhoria da sua atuação. Todas as entrevistas foram realizadas nas unidades de saúde, em uma sala reservada, com duração média de 30 minutos. Os relatos foram gravados, transcritos e analisados de acordo com a técnica de análise do Discurso do Sujeito Coletivo (DSC) (LEFEVRE et al., 2003). Foram considerados: representações, crenças e valores de uma coletividade sobre determinados temas, levando em consideração o universo de significados, motivos, aspirações, composição do território; relações sociais interpessoais da comunidade, de grupos, de classes, de gênero, ou objetivadas em instituições e rede de acesso aos serviços fora da comunidade.

\section{Violência urbana e saúde: algumas dimensões do problema}

A violência em meio urbano e suas consequências constituem um tema que vem ocupando cada vez mais espaço nas agendas públicas de saúde. A relevância dos efeitos diretos e indiretos das dinâmicas da violência sobre o setor saúde provocou o crescente interesse de especialistas nas últimas duas décadas, seja pelas consequências da 
violência para a saúde das populações mais gravemente afetadas, seja pelo impacto sobre o processo de trabalho dos serviços de saúde (MINAYO, 1994, 2006).

Segundo definição da Organização Mundial da Saúde, a violência é o uso intencional da força física ou poder, de forma real ou sob a forma de ameaça, contra uma pessoa, um grupo ou comunidade, que resulta ou tem grande possibilidade de resultar em lesão, morte, dano psicológico, baixo desenvolvimento ou privação (KRUG et al., 2002). No conjunto dos efeitos diretos da violência, o número de homicídios é considerado indicador universal e utilizado como parâmetro de mensuração e de comparação entre regiões ou países, sendo atualmente o principal responsável pelos elevados índices de mortalidade relacionados à violência na população mundial (UNITED NATIONS OFFICE ON DRUGS AND CRIME [UNODC], 2011). Segundo estudo de prospecção da Organização Mundial da Saúde (OMS) para identificação da causa de mortalidade em 2002, foram computados, no conjunto de países participantes, em torno de 520.000 homicídios, uma taxa de 8,8/100 mil habitantes (KRUG et al., 2002).

Assim como ocorre com outros agravos, as taxas de homicídios não se distribuem de forma homogênea. $O$ levantamento da OMS revelou que as taxas de homicídio são significativamente superiores na América Latina (19,9/100 mil hab.) e Caribe (16,3/100 mil hab.), se comparadas aos outros continentes, África (10,1/100 mil hab.), América da Norte (5,6/100 mil hab.), Ásia (2,1/100 mil hab.) e Europa (1,2/100 mil hab.). O estudo revela ainda que, ao se analisarem as taxas de homicídio para o recorte da população juvenil nos 83 países pesquisados, os números são muito superiores às médias gerais, sendo possível identificar 5 países latino-americanos nas 5 primeiras posições: El Salvador (92,3/100 mil hab.), Colômbia (73,4/100 mil hab.), Venezuela (64,2/100 mil hab.), Guatemala (55,4/100 mil hab.) e Brasil (51,6/100 mil hab.). Em recente estudo multicêntrico sobre mortalidade por homicídios em 4 países da América Latina, Souza, E. et al. (2012) identificaram a maior incidência de jovens do sexo masculino, seja como autores seja como vítimas da violência, e o uso predominante das armas de fogo para perpetrar os homicídios.

Neste sentido, o contexto sóciodemográfico, os indicadores de desigualdades sociais, e por consequência, a marginalização de determinados segmentos populacionais são fatores preponderantes para a compreensão da complexidade multicausal do fenômeno da violência (PORTO, 2000; WACQUANT, 2001). Além disso, considera-se que fatores históricos favorecem e cronificam efeitos estruturais de desigualdade (MINAYO, 2006), onde o fenômeno da violência se integra à organização das relações sociais (MESSARI; SOARES, 2008).

\section{Violência na Comunidade: um olhar para o território}

As diversas regiões de uma grande cidade são territórios particulares, e por vezes, desagregados um dos outros, com suas histórias sociais, políticas e, sobretudo, de ocupação dos espaços. São constituídos de modos variados, múltiplos, e às vezes discrepantes. O território considerado neste artigo faz parte de uma das comunidades mais populosas do município do Rio de Janeiro. Uma área marcada por uma série de situações de violência, onde a disputa pelo mercado de drogas é a principal causa dos confrontos armados. A evolução histórica da trajetória desta comunidade revela intervenções sistemáticas do Estado, marcadas por embates, contradições, demolições e desapropriações. Notadamente, a comunidade mostra-se dividida entre um núcleo mais urbanizado, que concentra as principais atividades de comércio e serviços, e um entorno mais carente, onde reside a população de menor poder aquisitivo.

A população estimada é de 140 mil habitantes. Segundo dados do Instituto Brasileiro de Geografia e Estatística [IBGE] (2010), os números de habitantes e de domicílios, no Conjunto de Favelas da Maré por comunidade, são respectivamente, 136.474 e 43.499. O Índice de Desenvolvimento Humano (IDH) é um dos mais baixos, 0.722 , contra a média de 0.845 da cidade. ${ }^{2} \mathrm{O}$ índice de educação é o segundo pior de todo estado e a expectativa de vida no local é de 66,58 anos, contra 79,47 no bairro do Leblon, por exemplo. A área conta com oito unidades de saúde, totalizando 34 equipes de atenção básica.

A pluralidade interna é um dos atributos do território. Ao conhecer melhor a comunidade, é possível observar que as várias localidades que a compõem foram construídas em diferentes condições históricas e políticas, o que se refletiu em uma diversidade de hábitos e costumes. A estética da área mais desenvolvida é algo que chama a atenção, composta por vias amplas, bem asfaltadas e cheias de quebra-molas. Destaca-se um comércio muito farto, vários mercados, lojas de móveis, barraquinhas de frutas, bares, muitas vans, moto-táxis, e muitas pessoas circulando.

Em cada esquina pode ser visto um "soldado do tráfico", vigilante a tudo que acontece no local. A circulação de pessoas é livre, desde que devidamente "autorizada pelo tráfico". As rivalidades constantes entre as facções, na tentativa de assegurar o monopólio da venda de drogas, obrigam a população a sujeitar-se à presença de homens armados, violência física, uso de drogas e "bocas de fumo"3 em suas calçadas. A presença armada do tráfico é ostensiva, principalmente nos finais de tarde ou nos fins de semana; jovens exibem fuzis, carros de luxo, cordões de ouro e motos importadas.

A disputa entre as facções rivais pelo controle do tráfico de drogas cria uma dinâmica social particular, marcada, sobretudo, por uma atmosfera de temor e pela imposição de leis próprias. Igualmente, as incursões policiais produzem uma atmosfera de medo. E, para além do entendimento de que possíveis ações pontuais da polícia traria a percepção de resolubilidade esperada, tem algo do histórico que foi construído na comunidade local, que produz

${ }^{2}$ Fonte: Instituto Brasileiro de Geografia e Estatística (IBGE, 2010). A população residente em Mandacaru, na comunidade de Marcílio Dias, foi removida após o Censo do IBGE. Aproximadamente, 1.600 pessoas residiam naquela porção da comunidade.

${ }^{3}$ Local onde a droga é vendida. 
amedrontamento toda vez que se ouve falar que haverá uma ação policial na comunidade, o que traz a ideia de que a polícia entra na comunidade para matar e desrespeitar.

Ficamos sozinhos, ouvimos e vemos coisas que não sabemos o que fazer. Um dia vi grupo de traficantes baterem em um menino de uns 14 anos, não aguentei e pedi para eles pararem [...] eles não pararam e deram um tiro no menino bem na minha frente. O que posso fazer com isso? Chamar a polícia? Vocês acham que eles não sabem o que se passa aqui dentro? (Agente Comunitário de Saúde [ACS]).

Fui humilhada por morar na Maré [...] a polícia entrou, me revistou e perguntou o porquê de estar tão nervosa, que se eu valesse alguma coisa, não estaria morando na favela [...] cresci vendo a polícia bater na cara das pessoas e entrar atirando [...], minha filha de 2 anos, vê um policial e sai correndo de medo [...] (Entrevista narrativa; ACS).

A compreensão deste contexto sociocultural é fundamental para entender os hábitos e costumes dos moradores, e como estes se apropriam do território e se relacionam com os serviços de saúde.

\section{Violência urbana e saúde mental: vulnerabilidades específicas?}

A caracterização da comunidade permite compreender uma dimensão pouco visível do impacto da violência, suas consequências psicossociais, aquelas que não se referem ao número de mortos e feridos, mas que produzem impactos subjetivos importantes para usuários e profissionais dos serviços de saúde. A violência urbana nas grandes cidades, sua dimensão armada, suas principais consequências e, sobretudo, as possibilidades de intervenção ainda são pouco debatidas no âmbito das estratégias assistenciais do setor saúde. No que se refere ao campo da atenção psicossocial, o cenário não é diferente. Apesar de alguns autores apontarem indícios de alta prevalência de transtorno mentais associados a eventos traumáticos em grandes cidades (MARI; MELLO; FIGUEIRA, 2008), há ausência de sistematização de conhecimento acerca das dimensões do problema e das possibilidades de intervenção no campo da saúde mental (DELGADO, 2012). Os resultados preliminares desta pesquisa vêm demonstrando, ainda que de forma incipiente, elementos que contribuem para um desenho sobre as implicações da violência armada em meio urbano para o campo psicossocial. Neste sentido, propõe-se como hipótese que o campo das vulnerabilidades psicossociais relacionadas com o cenário da violência urbana pode ser organizado em três dimensões principais, descritas a seguir: a violência enquanto agravo, barreira e risco.

\section{Aspectos clínicos: a violência enquanto agravo}

A primeira dimensão do problema se refere aos aspectos clínicos, ou seja, os agravos de natureza psicopatológica relacionados com a exposição a uma ou a várias experiências de violência. Em relação a esta dimensão, alguns estudos epidemiológicos vêm apontando elevada incidência de sintomas sugestivos de transtornos mentais, tais como transtornos depressivos, quadros de

Fractal, Rev. Psicol., v. 29 - n. 1, p. 17-23, 2017 ansiedade (que inclui o Transtorno de Estresse Pós-Traumático- TEPT) e outros quadros difusos, relacionados à dinâmica da violência armada em territórios vulneráveis.

As pesquisas que relacionam experiências de violência à incidência de transtorno mental não são conclusivas, mas demonstram algumas pistas importantes. Uma delas refere-se, por exemplo, ao fato de os estudos apontarem que os homens estão mais expostos aos eventos de violência que as mulheres, mas são elas que desenvolvem mais problemas de saúde mental quando vivenciam experiências de violência (RIBEIRO et al., 2009). No que se refere às experiências de assistência às pessoas afetadas por situações de violência, relatos sistemáticos vêm sendo produzidos a partir das intervenções humanitárias internacionais. Essas experiências de suporte psicossocial em situações de violência situam-se no contexto das emergências, sejam elas relacionadas a conflitos armados, ${ }^{4}$ seja nos cenários das catástrofes naturais e industriais. Em revisão sistemática recente sobre essas experiências, Tol et al., (2011) identificaram, junto a populações afetadas por conflitos armados, índices médios de prevalência de TEPT e depressão muito superiores àqueles encontrados nas 17 populações participantes do survey mundial de saúde mental da OMS (THE WHO WORLD MENTAL HEALTH SURVEY CONSORTIUM, 2004).

A dinâmica da violência nos territórios urbanos se diferencia daquelas encontradas nos cenários emergenciais das intervenções humanitárias internacionais. Além disso, a escassez de estudos que associam prevalência de transtornos mentais às situações de violência em territórios urbanos vulneráveis coloca obstáculos importantes à caracterização das especificidades dos agravos clínicos. $\mathrm{O}$ mais visível destes obstáculos se refere à própria definição de evento traumático, uma vez que, em geral, nos referimos a populações historicamente expostas a eventos de violência, como o testemunho de execuções sumárias, agressões físicas, confrontos armados e imposição da ordem pela via de ameaças. Os relatos dos agentes comunitários de saúde (ACS), da comunidade pesquisada, ilustram bem esse cotidiano:

Uma vez fui fazer uma visita domiciliar com uma colega, e o tiroteio começou, os bandidos começaram a correr atirando, caíam baleados na nossa frente, o caveirão entrou dando tiro para todos os lados, e nós duas ali no meio disso tudo. Nessa hora eu fiquei muito nervosa e foi a colega quem me ajudou; se estivesse sozinha, nem sei o que eu iria fazer na hora. As pessoas fecham as portas de suas casas; se elas te conhecem, elas até deixam você entrar, mas se não te conhecem você fica no meio da guerra! (Entrevista narrativa; ACS).

Também no campo dos agravos clínico-situacionais da violência urbana, podemos incluir as vulnerabilidades específicas dos portadores de transtornos mentais graves (esquizofrenia e outros quadros psicóticos), onde a exposição a confrontos e à rotina de ameaças agravam significativamente seus quadros clínicos. Além disso, há

${ }^{4}$ Como referência sobre experiências de suporte psicossocial em contextos de conflitos armados, ver as edições da revista Intervention, the Journal of Mental Health \& Psychosocial Support in Conflict Affected Areas. 
relatos sobre pessoas com transtornos mentais graves que são frequentemente agredidos, ou até assassinados no interior das comunidades, em função de pequenos furtos, ou por conta de algum comportamento "ameaçador" ou "estranho". ${ }^{5}$ Em outras situações, usuários dos Centros de Atenção Psicossocial (CAPS) são cooptados para pequenos "serviços" ao narcotráfico ou têm acesso livre ao consumo de drogas, principalmente crack e cocaína, por vezes em troca de suas próprias medicações psicotrópicas (SOUZA, F. et al., 2011).

\section{Acesso aos serviços de saúde mental: a violência enquanto barreira}

O termo violência urbana revela diferentes significados e consequências. Trata-se de uma construção social com demarcações temporais e espaciais. Toda violência tem continuamente um contexto, o qual corresponde não apenas aos atores ou vítimas, mas conjuntamente àqueles que a percebem. Eventos de violência urbana podem ocorrer em distintos lugares, mas cada um está capturado por seu contexto espacial, ou seja, existe uma apropriação própria dependendo do seu contexto (LAWRENCE; KARIM, 2007). A esta questão está associada à segunda dimensão de impacto da dinâmica da violência urbana para o campo de atenção psicossocial, que diz respeito à sua face de barreira de acesso aos serviços de saúde mental. Obviamente, o problema não se refere apenas aos serviços de saúde mental, mas, ao mesmo tempo, impõe a este campo elementos específicos de vulnerabilidade. Os relatos de profissionais que atuam em ambulatórios e nos CAPS, que em geral ficam fora dos territórios afetados diretamente pela violência, repetem histórias de usuários que faltam aos atendimentos em função de conflitos na comunidade. Com isso, a remarcação das consultas e os prejuízos para o tratamento são inevitáveis.

Invariavelmente, o contexto de violência em algumas comunidades e as "regras" de acesso impostas pelo tráfico de drogas limitam muito a entrada de profissionais de saúde de fora da comunidade, mesmo que devidamente identificados. Com isso, a visita domiciliar da equipe do CAPS, um potente recurso de cuidado, fica comprometida. A mesma restrição é imposta às ambulâncias, para os casos onde há necessidade de internação. Em alguns territórios, os serviços de remoção se recusam a entrar, não restando às famílias outra possibilidade que não seja levar, com seus próprios meios, o paciente em crise ao atendimento psiquiátrico de urgência (CORREA; SOUZA, S., 2011).

\section{Efeitos para os profissionais de saúde: a violência enquanto risco}

As barreiras de acesso e os riscos presentes nos territórios em situações de violência urbana afetam não apenas os usuários e suas famílias, mas também os profissionais dos serviços, sejam eles técnicos dos CAPS, sejam as equipes de atenção primária que trabalham sob essa condição. Neste sentido, os atores principais con-

\footnotetext{
Para melhor compreensão acerca da complexidade e manejo de agravos envolvendo portadores de transtornos mentais em situações de violência urbana, ver a cartilha "O cuidado ajuda a reatar laços" (COMITÊ INTERNACIONAL DA CRUZ VERMELHA [CICV], 2013).
}

siderados no estudo foram os ACS, já que estes profissionais têm uma especificidade no trabalho, pois fazem parte dessa população e trabalha para ela, com ela; supõe-se que o ACS é quem conhece as formas cotidianas de viver, de significar a vida e de se comportar das famílias locais. Vale ressaltar que, diante das necessidades, os moradores da comunidade buscam em primeiro lugar o ACS, quer seja para uma informação, uma reclamação ou a solução de um problema mais grave, e dele esperam uma resposta, em uma relação de cobranças e exigências nem sempre tranquila. Por este motivo, a opção é concentrar a pesquisa na visão e no sofrimento específico do ACS, baseada na quantidade e na intensidade de estímulos a que este é submetido e nos muitos recursos que dele são exigidos, a fim de concretizar a vigilância e atenção à saúde do território sob sua responsabilidade.

No que se refere aos profissionais de atenção primária, a frequência diária no território representa, ao mesmo tempo, um fator de proteção, na medida em que passam a ser conhecidos pela comunidade, e um fator de risco, por conta da maior exposição ao cenário de violência. São frequentes os relatos dos ACS acerca da necessidade de conhecimento dos 'sinais' do território, como medida de segurança.

Outro fator de risco refere-se à própria rotina de atendimento, onde esses profissionais têm contato cotidiano com o sofrimento dos seus usuários, em função do cenário de violência. Alguns autores nomeiam os efeitos secundários do atendimento a vítimas de violência como trauma vicário (SCHESTATSKY et al., 2003) ou trauma secundário (CROTHERS, 1995).

No contexto dos riscos, vale destacar a condição específica de vulnerabilidade do ACS. São frequentes as falas dos ACS que exprimem sentimentos de impotência frente às situações de precariedade, o não reconhecimento dos esforços realizados e a dificuldade no estabelecimento de fronteiras entre os aspectos profissionais e pessoais. Quando estes fatores se somam ao convívio próximo às situações de violência, como o cotidiano de intermediação com as leis do tráfico e incertezas de segurança do território, evidencia-se um aumento desta vulnerabilidade:

Eu me sinto incapaz de mudar alguma coisa, já vi algumas pessoas sendo mortas. Um dia vi um rapaz que assaltou um ônibus na Zona Sul, ele era morador da comunidade e foi reconhecido pelo motorista que fez a denúncia para os traficantes. Quando isso acontece os traficantes fazem julgamento em praça pública (entrevista narrativa; ACS).

$\mathrm{Na}$ tentativa de reduzir sua exposição aos riscos, os ACS traçam estratégias de proteção, como, por exemplo, evitar falar de situações comprometedoras, principalmente em grupo, e são discretos quanto à falta de segurança do território.

É possível observar, por fim, que as situações de risco para os profissionais de atenção primária produzem, por consequência, rotatividade de técnicos nos serviços, o que acarreta descontinuidade nos processos de trabalho e enfraquecimento da construção de vínculos entre os profissionais e os usuários. Pode-se identificar, portanto, 
uma condição de risco duplo, uma vez que a dinâmica de violência afeta diretamente os profissionais e, ao mesmo tempo, o processo de implementação das estratégias de atenção primária em saúde nestes territórios:

Quando novos profissionais chegaram, pensamos, puxa vida, tomara que eles não vão embora como os outros; só o fato de eles estarem aqui, já nos sentimos mais seguros, sabemos que se precisarmos podemos procurá-los. Mas fico pensando como eles conseguem entrar aqui? Como fazem para entrar? (Entrevista narrativa; ACS).

No que se refere ao recorte das estratégias psicossociais na atenção primária, a alta rotatividade de profissionais (decorrente não só do contexto de violência, mas atribuível também à precarização do trabalho no SUS, no município do Rio de Janeiro) produz um impacto ainda mais grave, uma vez que o êxito dessas estratégias está diretamente relacionado com a qualidade dos vínculos entre usuários e seus técnicos de referência. $\mathrm{O}$ atendimento prestado nas unidades básicas de saúde é referência para os moradores da comunidade e se as regras do tráfico impõem limites de circulação quando, por exemplo, moradores de um determinado território são 'impedidos' de ir a determinadas áreas da comunidade de outra facção, a mobilidade dos profissionais de saúde também fica limitada pela violência, o que contribui para precarização da assistência, comprometendo a proposta do SUS.

\section{Atenção Básica em contextos de violência: caminhos de uma agenda para a Saúde Mental?}

Novas propostas vêm surgindo na busca de reverter o modelo hospitalocêntrico e possibilitar a implementação efetiva do SUS. Como medida de gerenciar e acolher as diversas demandas de saúde e suas especificidades, a ESF passa a incluir profissionais de várias especialidades. Assim, no ano de 2008, com o objetivo de aumentar a abrangência e a resolubilidade da ESF, o Ministério da Saúde criou os Núcleos de Apoio à Saúde da Família (NASF). O NASF deve ser constituído por equipes compostas por profissionais de diferentes áreas de conhecimento, para atuarem em conjunto, "num processo de construção compartilhada" com os profissionais das Equipes Saúde da Família (CHIAVERINI, 2011, p. 13).

O conceito de apoio matricial foi desenvolvido por Cunha e Campos (2011, p. 964), dentro de uma metodologia de cuidado voltada para "a reforma das organizações e do trabalho em saúde", e de seu "modelo de atenção e de gestão". Esse método de gestão do cuidado foi, inicialmente, adotado em serviços de saúde mental, de atenção básica e da área hospitalar do Sistema Único de Saúde de Campinas-SP, Belo Horizonte - MG e também na cidade de Rosário/Argentina. Assim, o apoio matricial em saúde delineia a retaguarda especializada a equipes e profissionais da estratégia de saúde da família, como zelador da atenção a problemas de saúde, de maneira singular e compartilhada.

Apesar de os princípios do SUS apontarem para efetiva ampliação das ações em saúde mental, as práticas na atenção primária exigem uma aliança entre dimensões po- líticas e sociais, e se apresentam nas comunidades, como um processo de trabalho, fundamentado na multiplicidade de elementos que constituem o trabalho em saúde.

No que se refere às estratégias psicossociais em favor das populações afetadas pela violência, um dos principais desafios para as equipes da ESF e NASF diz respeito à invisibilidade das situações de violência, como um fator de agravo à saúde dos usuários. Algumas hipóteses acerca dessa invisibilidade poderiam ser formuladas, sejam relacionadas à banalização dos efeitos da violência por parte dos usuários, sejam associadas a um distanciamento dos profissionais em relação às histórias de vida dos usuários, por vezes ricas de detalhes assustadores. Embora este artigo não tenha por objetivo investigar essas hipóteses, é possível verificar que o profissional de saúde mental do NASF é um elemento estratégico para retirar esse tema da invisibilidade e, por consequência, da falta de assistência (BONFIM, et al., 2013).

Algumas experiências das equipes da ESF, em parceria com os NASF, vêm demonstrando efeitos importantes, seja na identificação e manejo de casos onde os quadros de sofrimento psíquico têm relação com as situações de violência, seja nas estratégias de cuidado voltadas aos portadores de transtornos mentais graves. Alguns relatos demonstram, por exemplo, que a oferta de abordagens em grupo para pessoas em situação de violência vem produzindo resultados, tanto no que se refere à possibilidade de suporte através das equipes da ESF e NASF, quanto na construção de espaços de apoio mútuo entre os participantes (QUEIROZ; VILARIM, 2012).

Em alguns casos, em função das situações de risco, o tempo da intervenção é fundamental e as definições assistenciais precisam de respostas imediatas, como ilustra a história de Beto, ${ }^{6}$ um usuário que chegou à equipe através da agente comunitária de saúde:

[...] ele fica o dia todo na minha porta, falando coisas que não existem. Às vezes, tira a roupa e faz as "necessidades" ali mesmo. Ele não dorme, fica falando sozinho a noite toda, parece que está falando com alguém ou vendo coisas que não existem! [...] Fico com medo, pois não sei o que fazer com ele! [...] ele acabou perdendo sua casa, pois foi invadida por traficantes e vendida para outras pessoas e com isso perdeu toda sua documentação. A partir de então, passou a dormir na minha porta (Entrevista narrativa; ACS).

$\mathrm{O}$ relato foi trazido durante a atividade de matriciamento, que se seguiu de uma visita domiciliar com a equipe de referência. Beto estava em situação de rua, desde que perdeu os pais. Neste período teve diversas passagens pela delegacia por pequenos furtos. Ele faz uma construção delirante de que é "dono do tráfico da Favela" (sic) e possui vínculo com a ACS da área desde sua infância. Recentemente, em uma fase mais aguda de desorganização psíquica, Beto deixou de ir à unidade, fez pequenos furtos e começou a pegar roupas nas cordas dos moradores para vestir. Por conta disso, Beto foi ameaçado de morte caso continuasse na comunidade. A equipe do NASF, juntamente com os profissionais da ESF e do CAPS, prontamente teve que reformular as estratégias de ${ }^{6}$ Nome fictício 
cuidado para preservar a vida de Beto. Ele foi encaminhado à emergência psiquiátrica de referência e novos arranjos tiveram que ser feitos.

A partir da inserção do profissional do NASF, é possível levantar algumas hipóteses quanto às possibilidades de potencialização das estratégias de saúde mental no território. No que se refere à especificidade do contexto urbano, a principal contribuição diz respeito à possibilidade de retirar da invisibilidade os efeitos, diretos e indiretos, da dinâmica da violência. Como consequência deste desvelamento, há espaço para o incremento das estratégias de gestão compartilhada do cuidado com a ESF, que passam a contemplar atividades de (1) suporte aos usuários (individuais ou grupos), (2) apoio aos profissionais que atuam nestes contextos (sobretudo os ACS que são também moradores do território), (3) educação permanente junto às equipes para a abordagem de pessoas afetadas pela violência e (4) articulação com outros serviços de saúde e outras redes (educação, assistência, justiça etc.).

\section{Considerações finais}

Os efeitos psicossociais da violência nas grandes cidades, sobretudo para as populações mais gravemente afetadas, revelam um horizonte de necessidades e de possibilidades de suporte ainda a ser explorado e discutido. $\mathrm{O}$ reconhecimento das experiências dos agentes comunitários e dos saberes locais podem possibilitar maior integração entre todos os atores envolvidos no processo, bem como favorecer a descoberta de novas formas de relação, que sejam capazes de gerar o sentimento de pertencimento, necessário para o exercício do protagonismo comunitário e o cuidado em saúde mental. O desenvolvimento de estratégias psicossociais no território tem como vetor a ampliação da rede de atenção primária, mas coloca para esta rede desafios importantes. O principal deles refere-se à migração das estratégias de cuidado, antes restritas aos ambulatórios especializados, para o contexto do território, seja através de parcerias com os CAPS, seja com o apoio do NASF. Deste modo, as estratégias de cuidado em favor das pessoas afetadas pela violência, e também das equipes de atenção primária, necessitam ser desenvolvidas e aperfeiçoadas, incluindo a complexidade teórico-metodológica e a plasticidade operacional, que são atributos do campo da atenção psicossocial brasileiro. A pesquisa vem demonstrando que o fio condutor do trabalho em comunidades afetadas pela violência urbana é a valorização dos coletivos para o favorecimento de espaços de circulação da palavra, caminho a ser materializado em propostas que valorizem o saber local e as especificidades de cada comunidade.

\section{Referências}

BONFIM, I. G. et al. Apoio matricial em saúde mental na atenção primária à saúde: uma análise da produção científica e documental. Interface, Botucatu, v. 17, n. 45, p. 287-300 abr.jun. 2013. Disponível em <http://www.scielo.br/scielo. php?script=sci_arttext\&pid=S1414-32832013000200004>. Acesso em: 5 ago. 2013.

CHIAVERINI, D. H. (Org.). et al. Guia prático de matriciamento em saúde mental. Brasília: Ministério da Saúde, 2011.
COMITÊ INTERNACIONAL DA CRUZ VERMELHA. $O$ cuidado ajuda a reatar laços: cartilha sobre saúde mental e violência para os agentes comunitários de saúde. Rio de Janeiro: CICV, 2013. Disponível em: <http://saudementalrj. blogspot.com.br/2013/01/o-cuidado-ajuda-reatar-lacos.html> Acesso em: 3 mar. 2013.

CORREA, C. S.; SOUZA, S. J. Violência e vulnerabilidades: os jovens e as notícias de jornal. Fractal, Rev. Psicol., Niterói, v. 23, n. 3, p. 461-486, set./dez. 2011. Cross ${ }^{\text {Ref. }}$

CROTHERS D. Vicarious traumatization in the work with survivors of childhood trauma. Psychosoc Nurs Ment Health Serv, [S.1.], v. 33, n. 4, p. 9-13, 1995.

CUNHA, G. T.; CAMPOS, G. W. S. Apoio Matricial e Atenção Primária em Saúde. Saude soc. São Paulo, v. 20, n. 4, p. 961970, out./dez. 2011. Cross ${ }^{\text {Ref. }}$

DELGADO, P. G. G. Violência e saúde mental: os termos do debate. O Social em Questão, Rio de Janeiro, ano 15, n. 28; p.187-198, 2012. Disponível em: <http://osocialemquestao.ser. puc-rio.br/media/9artigo.pdf>. Acesso em: 12 dez. 2013.

INSTITUTO BRASILEIRO DE GEOGRAFIA E ESTATÍSTICA. IDH dos bairros da cidade do Rio de Janeiro. 2010. Disponível em: <http://www.wikirio.com.br/IDH_dos bairros_da_cidade_do_Rio_de_Janeiro> Acesso em: 1 ago. 2013.

INTERVENTION, THE JOURNAL OF MENTAL HEALTH \& PSYCHOSOCIAL SUPPORT IN CONFLICT AFFECTED AREAS. 2003. Disponível em: <http://www. interventionjournal.com>. Acesso em: 22 dez. 2013.

KRUG, E. G. et al. (Org.). Relatório mundial sobre violência e saúde. Genebra: Organização Mundial da Saúde, 2002.

LAWRENCE, B.; KARIM, A. (Ed.). On violence: a reader. Durham: Duke University Press, 2007.

LEFEVRE, A. M. C. et al. A utilização da metodologia do discurso do sujeito coletivo na avaliação qualitativa dos cursos de especialização Capacitação e Desenvolvimento de Recursos Humanos em Saúde-CADRHU. Saúde e Sociedade, São Paulo, v. 12, n. 2, p. 68-75, jul./dez. 2003.

MARI, J. J.; MELLO, M. F.; FIGUEIRA, I. The impact of urban violence on mental health. Rev. Bras. Psiquiatr. São Paulo: v. 30, n. 3, p. 183-184, 2008. Cross ${ }^{\text {Ref. }}$

MESSARI, N.; SOARES, L. E. Crime organizado, drogas, corrupção pública: observações comparativas sobre Argentina, Brasil, Colômbia, Guatemala, México e Venezuela. In: SORJ, B.; TIRONI, E. (Org.). Coesão social na América Latina: bases para uma nova agenda democrática. São Paulo: Instituto Fernando Henrique Cardoso, 2008. p. 02-39.

MINAYO, M. C. S. A violência social sob a perspectiva da saúde pública. Cad. Saúde Públ, Rio de Janeiro, v. 10, supl. 1, p. $07-18,1994$. Cross ${ }^{\text {Ref. }}$

MINAYO, M. C. S. Violência e saúde. Rio de Janeiro: Fiocruz, 2006.

PORTO, M. S. G. A violência entre a inclusão e a exclusão social. Tempo soc., São Paulo, v. 12, n. 1, p. 187-200, maio 2000. Cross ${ }^{\text {Ref. }}$ 
QUEIROZ, M. R.; VILARIM, A. Cuidado em saúde mental no contexto da violência armada em meio urbano: a invisibilidade do problema e os desafios para o matriciamento. In: COELHO, D. A. M. et al. (Org.). Sobre o início do matriciamento em saúde mental na cidade do Rio de Janeiro: reflexões, relatos e recomendações. Rio de Janeiro: Secretaria Municipal de Saúde e Defesa Civil - Subsecretaria de Atenção Primária - Vigilância e Promoção da Saúde - Coordenação de Saúde Mental, 2012. p. 50-52. Disponível em: <http://saudementalrj.blogspot. com.br/2012/12/sobre-o-inicio-do-matriciamento-em.html>. Acesso em: 3 set. 2013.

RIBEIRO, W. S. et al. Exposição à violência e problemas de saúde mental em países em desenvolvimento: uma revisão da literatura. Revista Brasileira de Psiquiatria, São Paulo, v. 31, supl. 2, p. 49-57, out. 2009. Cross ${ }^{\text {Ref. }}$

SCHESTATSKY S. et al. A vulnerabilidade do terapeuta e seu impacto sobre a função terapêutica. Rev. Bras. Psicoter., v. 5, n. 3, p. 307-317, set./dez. 2003.

SOUZA, E. R. et al. Estudo multicêntrico da mortalidade por homicídios em países da América Latina. Ciência \& Saúde Coletiva, Rio de Janeiro, v. 17, n. 12, p. 3183-3193, dez. 2012. Cross ${ }^{\text {Ref. }}$

SOUZA, F. M. et al. A Violência urbana e suas consequências em um centro de atenção psicossocial na zona norte do município do Rio de Janeiro. Saúde soc., São Paulo, v. 20, n. 2, p. 363-376, abr.jun. 2011. Cross ${ }^{\text {Ref. }}$

THE WHO WORLD MENTAL HEALTH SURVEY CONSORTIUM. Prevalence, severity, and unmet need for treatment of mental disorders in the World Health Organization World Mental Health Surveys. JAMA, [S.1.], v. 291, n. 21, p. 2581-2590, 2004. Cross ${ }^{\text {Ref }}$

TOL W. et al. Mental health and psychosocial support in humanitarian settings: linking practice and research. The Lancet, v. 378, p. 1581-1591, Oct. 2011. Cross ${ }^{\text {Ref. }}$

UNITED NATIONS OFFICE ON DRUGS AND CRIME. The 2001 Global Study on Homicide. Viena: UNODC; 2011.

WACQUANT, L. As prisões da miséria. Rio de Janeiro: J. Zahar, 2001.

Recebido em: 16 de janeiro de 2014

Aceito em: 23 de novembro de 2016 